Proceedings of the Edinburgh Mathematical Society (2007) 50, 477-492 (C)

DOI:10.1017/S0013091505001057 Printed in the United Kingdom

\title{
SOLUTIONS OF SECOND-ORDER INTEGRO-DIFFERENTIAL EQUATIONS ON PERIODIC BESOV SPACES
}

\author{
VERÓNICA POBLETE \\ Departamento de Matemática, Facultad de Ciencia, Universidad de Santiago de Chile, \\ Avenida Libertador Bernardo O'Higgins 3363, Santiago, Chile \\ (vpoblete@lauca.usach.cl)
}

(Received 7 July 2005)

\begin{abstract}
Maximal regularity for an integro-differential equation with infinite delay on periodic vectorvalued Besov spaces is studied. We use Fourier multipliers techniques to characterize periodic solutions solely in terms of spectral properties on the data. We study a resonance case obtaining a compatibility condition which is necessary and sufficient for the existence of periodic solutions.

Keywords: integro-differential equation; maximal regularity; Fourier multiplier; vector-valued periodic Besov space

2000 Mathematics subject classification: Primary 45N05

Secondary 45D05; 43A15
\end{abstract}

\section{Introduction}

We consider the following integro-differential equation with infinite delay:

$$
\left.\begin{array}{c}
u^{\prime \prime}(t)+\alpha u^{\prime}(t)=A u(t)+\int_{-\infty}^{t} c(t-s) A u(s) \mathrm{d} s+f(t), \quad 0 \leqslant t \leqslant 2 \pi, \\
u(0)=u(2 \pi), \\
u^{\prime}(0)=u^{\prime}(2 \pi),
\end{array}\right\}
$$

where $A$ is a closed linear operator defined on a Banach space $X, c \in L^{1}\left(\mathbb{R}_{+}\right)$is a scalar-valued kernel, $f$ is an $X$-valued function defined on $[0,2 \pi]$ and $\alpha$ is a real number.

We will study existence and uniqueness of solutions for (1.1) in the space of $2 \pi$-periodic vector-valued functions $B_{p q}^{s}(\mathbb{T} ; X)$ (Besov spaces), where $\mathbb{T}$ denotes the one-dimensional torus $\mathbb{R} / \mathbb{Z}$. Below, we briefly recall the definition of periodic Besov spaces in vector-valued case introduced in $[\mathbf{3}]$. For the scalar case, see [10, Chapter 9] and $[\mathbf{9}]$. An approach to periodic Besov spaces based on semigroup theory and abstract interpolation is presented in $[\mathbf{5}$, Chapter 4].

In this work we study directly the full problem (1.1) by a method based on operatorvalued Fourier multiplier theorems, which was initiated by Weis in $[\mathbf{1 2}]($ see also $[\mathbf{4}, \mathbf{1 1}])$ 
in the investigation of maximal regularity for abstract differential equations. Fourier multiplier theorems on $B_{p q}^{s}(\mathbb{T} ; X)$ were recently studied in $[\mathbf{3}]$ motivated by the maximal regularity of periodic solutions for the Cauchy problems of first and second order.

In this paper we are able to obtain a very simple characterization of maximal regularity for (1.1) only in terms of the boundedness of $\left\{d_{k}\left(b_{k}-A\right)^{-1}\right\}_{k \in \mathbb{Z}}$, where

$$
d_{k}=\frac{-k^{2}}{1+\tilde{c}(\mathrm{i} k)}, \quad b_{k}=\frac{\alpha \mathrm{i} k-k^{2}}{1+\tilde{c}(\mathrm{i} k)}
$$

and $\tilde{c}$ denotes the Laplace transform of $c$. We remark that the conditions that we impose on the kernel $c$ are satisfied by a large class of functions.

In the second part, we study a resonance case: we assume that there are $k_{1}, \ldots, k_{N} \in \mathbb{Z}$ such that $i k_{j}$ is a simple pole of $F(\lambda)=\left(\lambda^{2}+\alpha \lambda-(1+\tilde{c}(\lambda)) A\right)^{-1}$ for $j=1, \ldots, N$. In this case, we will show that equation (1.1) has a $B_{p, q}^{s}$-periodic strong solution if and only if $f$ satisfies suitable compatibility conditions (Theorem 4.3). Also in this case we give a representation formula for all the solutions, which allows us to study their regularity. We remark that a similar case was studied in $[\mathbf{7}]$ when $A$ generates an analytic semigroup, and in $[\mathbf{8}]$ in the case of first-order integro-differential equations for a general linear unbounded operator $A$. However, in [8] the resonance case was not considered. Our results extend those in [3, Theorem 5.3], where the case for $\alpha=0$ and $c \equiv 0$ was presented.

The paper is organized as follows. In $\S 2$, we recall some useful properties of Besov spaces and Marcinkiewicz's condition of second order to establish results on $B_{p q}^{s}$-Fourier multipliers. Section 3 is devoted to maximal regularity in $B_{p q}^{s}(\mathbb{T} ; X)$, where the appropriate notion of the strong solution is defined. In $\S 4$ we study the resonance case.

\section{Preliminaries}

Besov spaces form one class of function spaces that are of special interest. The relatively complicated definition is recompensed by useful applications to differential equations (see [1] for a concrete model).

Let $\mathcal{D}(\mathbb{T})$ be the space of all complex-valued infinitely differentiable functions on $\mathbb{T}$. The usual locally convex topology in $\mathcal{D}(\mathbb{T})$ is generated by the seminorms $\|f\|_{n}=$ $\sup _{t \in \mathbb{T}}\left\|f^{(n)}(t)\right\|$, where $n \in \mathbb{N} \cup\{0\}$. We let $\mathcal{D}^{\prime}(\mathbb{T} ; X):=\mathcal{B}(\mathcal{D}(\mathbb{T}) ; X)$. Elements in $\mathcal{D}^{\prime}(\mathbb{T} ; X)$ are called $X$-valued distributions on $\mathbb{T}$.

Let $\mathcal{S}$ be the Schwartz space on $\mathbb{R}$ and let $\Phi(\mathbb{R})$ be the set of all systems $\phi=\left\{\phi_{j}\right\}_{j \geqslant 0} \subset$ $\mathcal{S}$ satisfying

$$
\begin{aligned}
\operatorname{supp}\left(\phi_{0}\right) \subset[-2,2], & \\
\operatorname{supp}\left(\phi_{j}\right) \subset\left[-2^{j+1},-2^{j-1}\right] \cup\left[2^{j-1}, 2^{k+1}\right], \quad j \geqslant 1, & \\
& \sum_{j \geqslant 0} \phi_{j}(t)=1, \quad t \in \mathbb{R},
\end{aligned}
$$

and for $n \in \mathbb{N} \cup\{0\}$, there exists $C_{n}>0$ such that

$$
\sup _{j \geqslant 0, x \in \mathbb{R}} 2^{n j}\left\|\phi_{j}^{(n)}(x)\right\| \leqslant C_{n} .
$$


Let $1 \leqslant p, q \leqslant \infty, s \in \mathbb{R}$ and $\phi=\left(\phi_{j}\right)_{j \geqslant 0} \in \Phi(\mathbb{R})$. The $X$-valued periodic Besov spaces are defined by

$$
B_{p, q}^{s, \phi}(\mathbb{T} ; X)=\left\{f \in \mathcal{D}^{\prime}(\mathbb{T} ; X):\|f\|_{B_{p}^{s, q},}=\left(\sum_{j \geqslant 0} 2^{s j q}\left\|\sum_{k \in \mathbb{Z}} e_{k} \otimes \phi_{j}(k) \hat{f}(k)\right\|_{p}^{q}\right)^{1 / q}<\infty\right\},
$$

where, for $x \in X$, we denote by $e_{k} \otimes x$ the $X$-valued function $\left(e_{k} \otimes x\right)(t)=\mathrm{e}^{\mathrm{i} k t} x$.

We make the usual modification if $q=\infty$. Note also that the space $B_{\infty, \infty}^{s}$ is the familiar space of all Hölder continuous functions of index $s$ if $s \in(0,1)$.

We remark that the spaces $B_{p, q}^{s, \phi}$ are independent of $\phi \in \Phi(\mathbb{R})$, and the norms $\|\cdot\|_{B_{p, q}^{s, \phi}}$ are equivalent. We will simply denote $\|\cdot\|_{B_{p, q}^{s, \phi}}$ by $\|\cdot\|_{B_{p, q}^{s}}$ for some $\phi \in \Phi(\mathbb{R})$.

Remark 2.1. For some useful properties of $B_{p, q}^{s}(\mathbb{T} ; X)$ see [3, Theorem 2.3].

For a function $f \in B_{p q}^{s}(\mathbb{T} ; X), s>0$, denote by $\hat{f}(k)$, for $k \in \mathbb{Z}$, the $k$ th Fourier coefficient of $f$, that is,

$$
\hat{f}(k)=\frac{1}{2 \pi} \int_{0}^{2 \pi} \mathrm{e}^{-\mathrm{i} k t} f(t) \mathrm{d} t .
$$

Let $X$ and $Y$ be Banach spaces. We denote by $\mathcal{B}(X, Y)$ be the space of all bounded linear operators from $X$ to $Y$. When $X=Y$ we write simply $\mathcal{B}(X)$.

Definition 2.2. Let $X$ and $Y$ be Banach spaces and let $\left\{M_{k}\right\}_{k \in \mathbb{Z}} \subset \mathcal{B}(X, Y)$. We say that $\left\{M_{k}\right\}_{k \in \mathbb{Z}}$ is a $B_{p q}^{s}$-multiplier if, for each $f \in B_{p, q}^{s}(\mathbb{T} ; X)$ there exists $g \in B_{p, q}^{s}(\mathbb{T} ; Y)$ such that

$$
\hat{g}(k)=M_{k} \hat{f}(k) \text { for all } k \in \mathbb{Z} .
$$

In this case, it follows from the Closed Graph Theorem that there exist $C>0$ such that, for $f \in B_{p, q}^{s}(\mathbb{T} ; X)$, we have

$$
\left\|\sum_{k \in \mathbb{Z}} e_{k} \otimes M_{k} \hat{f}(k)\right\|_{B_{p, q}^{s}} \leqslant C\|f\|_{B_{p, q}^{s}} .
$$

Remark 2.3. Let $X, Y$ and $Z$ be Banach spaces. If $\left\{M_{k}\right\}_{k \in \mathbb{Z}} \subset \mathcal{B}(X, Y)$ and $\left\{N_{k}\right\}_{k \in \mathbb{Z}} \subset \mathcal{B}(Y, Z)$ are $B_{p q}^{s}$-multipliers, then $\left\{N_{k} M_{k}\right\}_{k \in \mathbb{Z}}$ is a $B_{p q}^{s}$-multiplier. This follows directly from the definition.

The following condition on sequences $\left\{M_{k}\right\}_{k \in \mathbb{Z}} \subset \mathcal{B}(X, Y)$ was introduced in $[2]$ to study Fourier multipliers in the $L^{p}$-context. It is also used in the study of multipliers of Besov spaces.

Definition 2.4. We say that a sequence $\left\{M_{k}\right\}_{k \in \mathbb{Z}} \subset \mathcal{B}(X, Y)$ is $M$-bounded if

$$
\begin{gathered}
\sup _{k \in \mathbb{Z}}\left\|M_{k}\right\|<\infty, \quad \sup _{k \in \mathbb{Z}}\left\|k\left(M_{k+1}-M_{k}\right)\right\|<\infty, \\
\sup _{k \in \mathbb{Z}}\left\|k^{2}\left(M_{k+1}-2 M_{k}+M_{k-1}\right)\right\|<\infty .
\end{gathered}
$$

The following general multiplier theorem is due to Arendt and $\mathrm{Bu}$ [3, Theorem 4.5].

Theorem 2.5. Let $X$ and $Y$ be Banach spaces and let $\left\{M_{k}\right\}_{k \in \mathbb{Z}} \subset \mathcal{B}(X, Y)$ be $M$ bounded. Then for $1 \leqslant p, q \leqslant \infty, s \in \mathbb{R},\left\{M_{k}\right\}_{k \in \mathbb{Z}}$ is a $B_{p q}^{s}$-multiplier. 


\section{Maximal regularity on $B_{p q}^{s}(\mathbb{T} ; X)$}

For a linear operator $A$ on $X$, we denote the domain by $D(A)$ and its resolvent set by $\rho(A)$, and for $\lambda \in \rho(A)$ we write $R(\lambda, A)=(\lambda I-A)^{-1}$.

We denote by $\tilde{c}$ the Laplace transform of $c \in L^{1}\left(\mathbb{R}_{+}\right)$. In what follows, we always assume that $\tilde{c}(\mathrm{i} k)$ exists for all $k \in \mathbb{Z}$ and that $\tilde{c}(\mathrm{i} k) \neq-1$ for all $k \in \mathbb{Z}$.

We adopt throughout the following notation:

$$
\begin{array}{ll}
d_{k}=\frac{1}{a_{k}}=\frac{-k^{2}}{1+\tilde{c}_{k}} & \text { for all } k \in \mathbb{Z} \backslash\{0\}, d_{0}=0, \\
b_{k}=\frac{\alpha \mathrm{i} k-k^{2}}{1+\tilde{c}_{k}} & \text { for all } k \in \mathbb{Z},
\end{array}
$$

where $\tilde{c}_{k}:=\tilde{c}(\mathrm{i} k)$.

Remark 3.1. Note that by the Riemann-Lebesgue lemma the sequences $\{\tilde{c}(\mathrm{i} k)\}$ and $\{1 /(1+\tilde{c}(\mathrm{i} k))\}$ are bounded.

Let $\left\{c_{k}\right\}_{k \in \mathbb{Z}} \subset \mathbb{C}$ be a sequence. We say that $\left\{c_{k}\right\}$ verifies the following conditions:

$$
\left\{k\left(c_{k+1}-c_{k}\right)\right\}_{k \in \mathbb{Z}} \text { and }\left\{k^{2}\left(c_{k+1}-2 c_{k}+c_{k-1}\right)\right\}_{k \in \mathbb{Z}} \text { are bounded. }
$$

Proposition 3.2. If $\{\tilde{c}(\mathrm{i} k)\}_{k \in \mathbb{Z}}$ verifies the condition (H1), then $\left\{a_{k}\right\}$ and $\left\{b_{k}\right\}$ defined by (3.1) and (3.2) verify that

$$
\left\{k a_{k}\left(b_{k+1}-b_{k}\right)\right\}_{k \in \mathbb{Z} \backslash\{0\}} \text { and }\left\{k^{2} a_{k}\left(b_{k+1}-2 b_{k}+b_{k-1}\right)\right\}_{k \in \mathbb{Z} \backslash\{0\}} \text { are bounded. }
$$

Proof. We have the identity

$$
k a_{k}\left(b_{k+1}-b_{k}\right)=\frac{-1}{1+\tilde{c}_{k+1}} k\left(\tilde{c}_{k+1}-\tilde{c}_{k}\right)-\frac{\alpha \mathrm{i}}{1+\tilde{c}_{k+1}}\left(\tilde{c}_{k}-\tilde{c}_{k+1}\right)+\frac{-2 k-1+\alpha \mathrm{i}}{k} \frac{1+\tilde{c}_{k}}{1+\tilde{c}_{k+1}} .
$$

By hypothesis and Remark 3.1 we obtain the first assertion of the proposition. In order to prove the assertion, we have the identity

$$
\begin{aligned}
& k^{2} a_{k}\left(b_{k+1}-2 b_{k}+b_{k-1}\right) \\
& =\frac{-1}{\left(1+\tilde{c}_{k+1}\right)\left(1+\tilde{c}_{k-1}\right)} \\
& \quad \times\left[\left(1+\tilde{c}_{k+1}\right) k^{2}\left(\tilde{c}_{k-1}-2 \tilde{c}_{k}+\tilde{c}_{k+1}\right)-k\left(\tilde{c}_{k+1}-\tilde{c}_{k-1}\right) k\left(\tilde{c}_{k+1}-\tilde{c}_{k}\right)\right. \\
& \quad+2\left(1+\tilde{c}_{k}\right) k\left(\tilde{c}_{k+1}-\tilde{c}_{k-1}\right)+\alpha \mathrm{i}\left(1+\tilde{c}_{k-1}\right) k\left(\tilde{c}_{k}-\tilde{c}_{k+1}\right) \\
& \quad+\alpha \mathrm{i}\left(1+\tilde{c}_{k+1}\right) k\left(\tilde{c}_{k}-\tilde{c}_{k-1}\right)+\alpha \mathrm{i}\left(1+\tilde{c}_{k}\right)\left(\tilde{c}_{k-1}-\tilde{c}_{k+1}\right) \\
& \left.\quad-\left(1+\tilde{c}_{k-1}\right)\left(1+\tilde{c}_{k}\right)-\left(1+\tilde{c}_{k+1}\right)\left(1+\tilde{c}_{k}\right)\right] .
\end{aligned}
$$

Hence, by hypothesis and Remark 3.1 we obtain the desired conclusion.

Proposition 3.3. Let $A$ be a closed linear operator defined on the Banach space $X$. Let $\left\{d_{k}\right\}_{k \in \mathbb{Z}}$ and $\left\{b_{k}\right\}_{k \in \mathbb{Z}}$ be defined by (3.1) and (3.2), respectively. Assume that $\left\{\tilde{c}_{k}\right\}_{k \in \mathbb{Z}}$ satisfies (H1). If $b_{k} \in \rho(A)$ for all $k \in \mathbb{Z}$ and $\left\{d_{k}\left(b_{k}-A\right)^{-1}\right\}_{k \in \mathbb{Z}}$ is bounded, then $\left\{d_{k}\left(b_{k}-A\right)^{-1}\right\}_{k \in \mathbb{Z}}$ is a $B_{p, q}^{s}$-multiplier, $1 \leqslant p \leqslant \infty$. 
Proof. Set $M_{k}=d_{k}\left(b_{k} I-A\right)^{-1}$. Note that $M_{0}$ is the null operator.

We will verify that the sequence $\left\{M_{k}\right\}$ is $M$-bounded. The result then follows from Theorem 2.5. In fact, first we prove (2.2). We have the identity

$$
k\left[M_{k+1}-M_{k}\right]=M_{k+1} k a_{k+1}\left[b_{k}-b_{k+1}\right] M_{k}+M_{k+1} k\left[1-\frac{a_{k+1}}{a_{k}}\right] .
$$

Note that

$$
\frac{a_{k+1}}{a_{k}}=\frac{1+\tilde{c}_{k+1}}{1+\tilde{c}_{k}}\left(\frac{k}{k+1}\right)^{2} .
$$

Hence, for each $k \in \mathbb{Z} \backslash\{-1\}$, we see that

$$
k\left[1-\frac{a_{k+1}}{a_{k}}\right]=\left[\frac{2 k^{2}+k}{(k+1)^{2}}+\frac{k^{2}}{(k+1)^{2}} \frac{1}{1+\tilde{c}_{k}} k\left(\tilde{c}_{k}-\tilde{c}_{k+1}\right)\right]
$$

is bounded, since $\left\{\tilde{c}_{k}\right\}$ verifies $(\mathrm{H} 1)$.

Moreover, for all $k \in \mathbb{Z} \backslash\{-1\}$, by Proposition 3.2 we find that $\left\{k a_{k+1}\left(b_{k}-b_{k+1}\right)\right\}$ is bounded. This, together with the boundedness of $\left\{M_{k}\right\}$, implies that

$$
\sup _{k \in \mathbb{Z}}\left\|k\left(M_{k+1}-M_{k}\right)\right\|<\infty .
$$

In order to verify condition (2.3), with an analogous calculation to that above we obtain

$$
\begin{aligned}
k^{2}\left(M_{k+1}-2 M_{k}+M_{k-1}\right)=k^{2} & \left(\frac{1}{a_{k+1}}-\frac{2}{a}+\frac{1}{a}{ }_{k-1}\right) a_{k+1} M_{k+1} \\
& -2 k\left[1-\frac{a_{k}}{a_{k-1}}\right] k a_{k-1}\left(b_{k+1}-b_{k}\right) M_{k} M_{k-1} \\
& -k^{2} a_{k}\left(b_{k+1}-2 b_{k}+b_{k-1}\right) M_{k} M_{k-1} \\
& +2 k a_{k+1}\left(b_{k+1}-b_{k}\right) k a_{k-1}\left(b_{k+1}-b_{k-1}\right) M_{k+1} M_{k} M_{k-1} \\
& -k a_{k}\left(b_{k+1}-b_{k}\right) k a_{k+1}\left(b_{k+1}-b_{k-1}\right) M_{k+1} M_{k} M_{k-1}
\end{aligned}
$$

where, with a direct calculation, we find that

$$
\begin{aligned}
k^{2}\left(\frac{1}{a_{k+1}}-\frac{2}{a_{k}}+\frac{1}{a_{k-1}}\right) a_{k+1}=\frac{k^{2}}{(k+} & 1)^{2}\left(1+\tilde{c}_{k}\right)\left(1+\tilde{c}_{k-1}\right) \\
\times[ & \left(1+\tilde{c}_{k+1}\right) k^{2}\left(\tilde{c}_{k+1}-2 \tilde{c}_{k}+\tilde{c}_{k-1}\right) \\
& +k\left(\tilde{c}_{k-1}-\tilde{c}_{k+1}\right) k\left(\tilde{c}_{k}-\tilde{c}_{k+1}\right) \\
& +2\left(1+\tilde{c}_{k}\right) k\left(\tilde{c}_{k-1}-\tilde{c}_{k+1}\right) \\
& \left.+\left(1+\tilde{c}_{k-1}\right)\left(1+\tilde{c}_{k}\right)+\left(1+\tilde{c}_{k+1}\right)\left(1+\tilde{c}_{k}\right)\right] .
\end{aligned}
$$

Since $\left\{\tilde{c}_{k}\right\}$ verifies (H1) we conclude that the sequence $\left\{k^{2}\left(1 / a_{k+1}-2 / a_{k}+1 / a_{k-1}\right) a_{k+1}\right\}$ is bounded for all $k \in \mathbb{Z} \backslash\{-1\}$. Hence, by Proposition 3.2 together with the boundedness of $\left\{M_{k}\right\}$, we find that $k^{2}\left(M_{k+1}-2 M_{k}+M_{k-1}\right)$ is bounded for all $k \in \mathbb{Z} \backslash\{-1,0,1\}$. Finally, since $M_{-2}, M_{2}, M_{-1}, M_{1}$ are well-defined operators we prove the claim. 
Lemma 3.4. Let $X$ be a Banach spaces. Assume that the Laplace transform $\left\{\tilde{c}_{k}\right\}_{k \in \mathbb{Z}}$ verifies condition (H1). Then the sequences $\left\{\left(1+\tilde{c}_{k}\right) I\right\}_{k \in \mathbb{Z}}$ and $\left\{1 /\left(1+\tilde{c}_{k}\right) I\right\}$ are $B_{p, q^{-}}^{s}$ multipliers.

Proof. It is clear, directly from (H1) and Theorem 2.5, that the sequence $\left\{m_{k}:=\right.$ $\left.\left(1+\tilde{c}_{k}\right) I\right\}$ is a $B_{p, q}^{s}$-multiplier.

Now, let $n_{k}:=1 /\left(1+\tilde{c}_{k}\right)$. The sequence $\left\{n_{k}\right\}$ is bounded and satisfies the identities

$$
k\left(n_{k+1}-n_{k}\right)=k\left[\tilde{c}_{k}-\tilde{c}_{k+1}\right] \frac{1}{1+\tilde{c}_{k}} \frac{1}{1+\tilde{c}_{k+1}}
$$

and

$$
\begin{aligned}
k^{2}\left(n_{k+1}-2 n_{k}+n_{k-1}\right)= & \frac{-1}{\left(1+\tilde{c}_{k}\right)} \frac{1}{\left(1+\tilde{c}_{k-1}\right)} k^{2}\left[\tilde{c}_{k+1}-2 \tilde{c}_{k}+\tilde{c}_{k-1}\right] \\
& +\frac{1}{1+\tilde{c}_{k+1}} \frac{1}{1+\tilde{c}_{k}} \frac{1}{1+\tilde{c}_{k-1}} k\left[\tilde{c}_{k+1}-\tilde{c}_{k-1}\right] k\left[\tilde{c}_{k+1}-\tilde{c}_{k}\right] .
\end{aligned}
$$

Hence, the sequence is $M$-bounded, proving the lemma.

Definition 3.5. Let $1 \leqslant p, q \leqslant \infty$ and $s>0$. A function $u \in B_{p, q}^{s+2}(\mathbb{T} ; X)$, is called a strong $B_{p, q}^{s}$-solution of (1.1) if $u(t) \in D(A)$ and (1.1) holds for almost every $t \in[0,2 \pi]$.

Theorem 3.6. Let $1 \leqslant p, q \leqslant \infty$ and $s>0$. Let $A$ be a closed linear operator defined on a Banach space $X$. If $\left\{\tilde{c}_{k}\right\}_{k \in \mathbb{Z}}$ satisfies (H1), then the following assertions are equivalent:

(i) $\left\{\frac{\alpha \mathrm{i} k-k^{2}}{1+\tilde{c}_{k}}\right\}_{k \in \mathbb{Z}} \subset \rho(A)$ and $\sup _{k}\left\|\frac{-k^{2}}{1+\tilde{c}_{k}}\left(\frac{-k^{2}+\alpha \mathrm{i} k}{1+\tilde{c}_{k}}-A\right)^{-1}\right\|<\infty$;

(ii) for every $f \in B_{p, q}^{s}(\mathbb{T} ; X)$, there exists a unique strong $B_{p, q}^{s}$-solution of (1.1) such that $u^{\prime \prime}, u^{\prime}, A u \in B_{p, q}^{s}(\mathbb{T} ; X)$.

Proof. (ii) $\Rightarrow$ (i). Let $x \in X$ be fixed. Define $f=e_{k} \otimes x$. Note that $f \in B_{p, q}^{s}(\mathbb{T} ; X)$. Hence, there exists $u \in B_{p, q}^{s+2}(\mathbb{T} ; X)$ such that $u(t) \in D(A)$ and (1.1) holds for almost every $t \in[0,2 \pi]$.

Taking Fourier transforms on both sides we obtain that $\hat{u}(k) \in D(A)$ and

$$
-k^{2} \hat{u}(k)+\alpha \mathrm{i} k \hat{u}(k)=A \hat{u}(k)+\tilde{c}_{k} A \hat{u}(k)+\hat{f}(k),
$$

where $\tilde{c}_{k}$ is the Laplace transform of $c$. Thus, $\left(-k^{2}+\alpha \mathrm{i} k-A-\tilde{c}_{k} A\right) \hat{u}(k)=\hat{f}(k)=x$, proving that $-k^{2}+\alpha \mathrm{i} k-A-\tilde{c}_{k} A$ is surjective.

Let $x \in D(A)$. If $\left(-k^{2}+\alpha \mathrm{i} k-A-\tilde{c}_{k} A\right) x=0$, that is $A x=\left(-k^{2}+\alpha \mathrm{i} k\right) I x /\left(1+\tilde{c}_{k}\right)$, then $u(t)=\mathrm{e}^{\mathrm{i} k t} x$ defines a periodic solution of

$$
u^{\prime \prime}(t)+\alpha u^{\prime}(t)=A u(t)+\int_{-\infty}^{t} c(t-s) A u(s) \mathrm{d} s .
$$


Hence, $u=0$ by the assumption of uniqueness, and thus $x=0$. Since $A$ is closed, by [6, Proposition 1.15] we conclude that

$$
\frac{\alpha \mathrm{i} k-k^{2}}{1+\tilde{c}_{k}} \subset \rho(A) \text { for all } k \in \mathbb{Z}
$$

Next we claim that

$$
\frac{-k^{2}}{1+\tilde{c}_{k}}\left(\frac{-k^{2}+\alpha \mathrm{i} k}{1+\tilde{c}_{k}}-A\right)^{-1}
$$

is a $B_{p, q}^{s}$-multiplier. Let $f \in B_{p, q}^{s}(\mathbb{T} ; X)$. By hypothesis, there exists a unique $u \in$ $B_{p, q}^{s+2}(\mathbb{T} ; X)$ such that

$$
u^{\prime \prime}(t)+\alpha u^{\prime}(t)=A u(t)+\int_{-\infty}^{t} c(t-s) A u(s) \mathrm{d} s+f(t) .
$$

Taking Fourier transforms of both sides, we find that $\hat{u}(k) \in D(A)$ and

$$
\hat{u}(k)=\left(-k^{2}+\alpha \mathrm{i} k-\left(1+\tilde{c}_{k}\right) A\right)^{-1} \hat{f}(k)
$$

or

$$
-k^{2} \hat{u}(k)=-\frac{k^{2}}{1+\tilde{c}_{k}}\left(\frac{-k^{2}+\alpha \mathrm{i} k}{1+\tilde{c}_{k}}-A\right)^{-1} \hat{f}(k) .
$$

By [3, Theorem 1.3], if $u \in B_{p, q}^{s+2}(\mathbb{T} ; X)$, then $u^{\prime}$ is differentiable almost everywhere (a.e.) and $u^{\prime \prime} \in B_{p, q}^{s}(\mathbb{T} ; X)$. Define $v=u^{\prime \prime}$. We then obtain

$$
\hat{v}(k)=-\frac{k^{2}}{1+\tilde{c}_{k}}\left(\frac{-k^{2}+\alpha \mathrm{i} k}{1+\tilde{c}_{k}}-A\right)^{-1} \hat{f}(k),
$$

proving the claim. It follows from the Closed Graph Theorem that there exist $C>0$ such that, for $f \in B_{p, q}^{s}(\mathbb{T} ; X)$, we have

$$
\left\|\sum_{k \in \mathbb{Z}} e_{k} \otimes M_{k} \hat{f}(k)\right\|_{B_{p, q}^{s}} \leqslant C\|f\|_{B_{p, q}^{s}} .
$$

Let $x \in X$ and define $f(t)=e_{n} \otimes x$ for $n \in \mathbb{Z}$ fixed. Then the above inequality implies that $\left\|e_{n}\right\|_{B_{p, q}^{s}}\left\|M_{n} x\right\|=\left\|e_{n} M_{n} x\right\| \leqslant C\left\|e_{n}\right\|_{B_{p, q}^{s}}\|x\|$. Hence, $\left\|M_{n}\right\| \leqslant C$.

(i) $\Rightarrow$ (ii). Let

$$
M_{k}=-\frac{k^{2}}{1+\tilde{c}_{k}}\left(\frac{-k^{2}+\alpha \mathrm{i} k}{1+\tilde{c}_{k}}-A\right)^{-1} .
$$

By assumption we see that $\left\{M_{k}\right\}_{k \in \mathbb{Z}}$ is a bounded sequence. We define

$$
N_{k}=\frac{1}{1+\tilde{c}_{k}}\left(\frac{-k^{2}+\alpha \mathrm{i} k}{1+\tilde{c}_{k}}-A\right)^{-1} .
$$

First, we claim that the families $\left\{\mathrm{i} k N_{k}\right\}_{k \in \mathbb{Z}}$ and $\left\{N_{k}\right\}_{k \in \mathbb{Z}}$ are $B_{p q}^{s}$-multipliers. In order to see this, we will apply Theorem 2.5 . 
In fact, in order to verify the condition (2.2), observe that $\left\|\mathrm{i} k N_{k}\right\| \leqslant\left\|k^{2} N_{k}\right\|=\left\|M_{k}\right\|$ for all $k \in \mathbb{Z}$ and hence that $\sup _{k \in \mathbb{Z}}\left\|\mathrm{i} k N_{k}\right\|<\infty$.

Moreover, we have the identity

$$
k\left[(k+1) N_{k+1}-k N_{k}\right]=-M_{k+1}+M_{k}-(k+1) N_{k+1},
$$

and hence the condition (2.2) holds, since $\left\{M_{k}\right\}$ is bounded.

To verify the condition (2.3), note that

$$
\begin{aligned}
& k^{2}\left[(k+1) N_{k+1}-2 k N_{k}+(k-1) N_{k-1}\right] \\
= & k\left[M_{k}-M_{k+1}\right]+k\left[M_{k}-M_{k-1}\right]-k\left[(k+1) N_{k+1}-k N_{k}\right]+k\left[(k-1) N_{k-1}-k N_{k}\right] .
\end{aligned}
$$

Since $\sup _{k \in \mathbb{Z}}\left\|M_{k}\right\|<\infty$, from the proof of Proposition 3.3 we see that the sequence $\left\{M_{k}\right\}$ verifies the condition (2.2) of Definition 2.4. Using this in the above identity, we conclude that the condition (2.3) holds for $\left\{\mathrm{i} k N_{k}\right\}$. We have the claim.

Second, we will prove that $\left\{N_{k}\right\}$ is a $B_{p q}^{s}$-multiplier. In fact, to verify the condition (2.2) observe that $\left\|N_{k}\right\| \leqslant\left\|k^{2} N_{k}\right\|=\left\|M_{k}\right\|$ for all $k \in \mathbb{Z} \backslash\{0\}$ and hence that $\sup _{k \in \mathbb{Z}}\left\|N_{k}\right\|<$ $\infty$. Moreover, we have

$$
k\left[N_{k+1}-N_{k}\right]=(k+1) N_{k+1}+N_{k}-N_{k+1},
$$

and since $\left\{k N_{k}\right\}$ and $\left\{N_{k}\right\}$ are bounded sequences we obtain condition (2.2).

In order to verify the condition (2.3), note that

$$
\begin{aligned}
k^{2}\left[N_{k+1}-\right. & \left.2 N_{k}+N_{k-1}\right] \\
& =-M_{k+1}+2 M_{k}-M_{k-1}-(k+1) N_{k+1}+(k-1) N_{k-1}+N_{k-1}-N_{k+1},
\end{aligned}
$$

and, since $\left\{M_{k}\right\},\left\{k N_{k}\right\}$ and $\left\{N_{k}\right\}$ are bounded sequences, we obtain condition (2.3) and the claim follows.

Now, let $f \in B_{p, q}^{s}(\mathbb{T} ; X)$. Since $\left\{N_{k}\right\}$ is a $B_{p q}^{s}$-multiplier, there exists $u \in B_{p, q}^{s}(\mathbb{T} ; X)$ such that

$$
\hat{u}(k)=N_{k} \hat{f}(k) \text { for all } k \in \mathbb{Z},
$$

where we observe that $\hat{u}(k) \in D(A)$.

Since $\left\{\mathrm{i} k N_{k}\right\}$ is a $B_{p q}^{s}$-multiplier, there exists $v \in B_{p, q}^{s}(\mathbb{T} ; X)$ such that $\hat{v}(k)=\mathrm{i} k N_{k} \hat{f}(k)$ for all $k \in \mathbb{Z}$. From (3.3) we obtain

$$
\mathrm{i} k \hat{u}(k)=\hat{v}(k) .
$$

By $\left[\mathbf{2}\right.$, Lemma 2.1], $u$ is differentiable a.e. with $u^{\prime}=v$ and $u(0)=u(2 \pi)$. By [3, Theorem 2.3 ] this implies that $u \in B_{p, q}^{s+1}(\mathbb{T} ; X)$.

Since $\left\{M_{k}\right\}$ is a $B_{p q}^{s}$-multiplier, there exists $w \in B_{p, q}^{s}(\mathbb{T} ; X)$ such that $\hat{w}(k)=M_{k} \hat{f}(k)$ for all $k \in \mathbb{Z}$. Again using the equalities (3.3) and (3.4), we have

$$
-k^{2} \hat{u}(k)=\mathrm{i} k \hat{v}(k)=\hat{w}(k) .
$$

By $[\mathbf{2}, \S 6] u^{\prime}$ is differentiable a.e. with $w=u^{\prime \prime}, u^{\prime}(0)=u^{\prime}(2 \pi)$ and $w=v^{\prime}=u^{\prime \prime}$. By $\left[\mathbf{3}\right.$, Theorem 2.3] this implies that $u \in B_{p, q}^{s+2}(\mathbb{T} ; X)$. 
We show that $u(t) \in D(A)$. By (3.3), we have the identity

$$
\left(-k^{2}+\alpha \mathrm{i} k-\left(1+\tilde{c}_{k}\right) A\right) \hat{u}(k)=\hat{f}(k)
$$

for all $k \in \mathbb{Z}$, or, equivalently,

$$
\begin{aligned}
A \hat{u}(k) & =\frac{-k^{2}+\alpha \mathrm{i} k}{1+\tilde{c}_{k}} \hat{u}(k)-\frac{1}{1+\tilde{c}_{k}} \hat{f}(k) \\
& =\frac{1}{1+\tilde{c}_{k}} \hat{w}(k)+\frac{\alpha}{1+\tilde{c}_{k}} \hat{v}(k)-\frac{1}{1+\tilde{c}_{k}} \hat{f}(k) .
\end{aligned}
$$

Since $f, v, w \in B_{p, q}^{s}(\mathbb{T} ; X)$ and by Corollary 3.4 the family $\left\{I /\left(1+\tilde{c}_{k}\right)\right\}$ is a $B_{p, q}^{s}$-multiplier, there exists $g \in B_{p, q}^{s}(\mathbb{T} ; X)$ such that

$$
A \hat{u}(k)=\hat{g}(k) \text {. }
$$

Then [2, Lemma 3.1] implies that $u(t) \in D(A)$ and $A u(t)=g(t)$. Hence, $A u \in B_{p, q}^{s}(\mathbb{T} ; X)$.

Finally, from (3.5), we have

$$
\left(-k^{2}+\alpha \mathrm{i} k\right) \hat{u}(k)=A \hat{u}(k)+A \tilde{c}_{k} \hat{u}(k)+\hat{f}(k) .
$$

Since $A$ is closed, from [2, Lemma 3.1] we deduce that

$$
u^{\prime \prime}(t)+\alpha u^{\prime}(t)=A u(t)+\int_{-\infty}^{t} c(t-s) A u(s) \mathrm{d} s+f(t) .
$$

It remains to show uniqueness. Let $u \in B_{p, q}^{s}(\mathbb{T} ; X)$ be such that

$$
u^{\prime \prime}(t)+\alpha u^{\prime}(t)-A u(t)-\int_{-\infty}^{t} c(t-s) A u(s) \mathrm{d} s=0 .
$$

Then $\hat{u}(k) \in D(A)$ and $\left[-k^{2}+\alpha \mathrm{i} k-\left(1+\tilde{c}_{k}\right) A\right] \hat{u}(k)=0$. Since

$$
\frac{-k^{2}+\alpha \mathrm{i} k}{1+\tilde{c}_{k}} \in \rho(A)
$$

this implies that $\hat{u}(k)=0$ for all $k \in \mathbb{Z}$ and thus that $u=0$.

In the case where $p=q=\infty$ and $0<s<1$ we find that $B_{\infty, \infty}^{s}(\mathbb{T} ; X)$ corresponds to the space $C^{s}(\mathbb{T} ; X)$ of Hölder continuous functions. We state the corresponding result.

Corollary 3.7. Let $0<s<1$. Let $A$ be a closed linear operator defined on a Banach space $X$. Assume that $\left\{\tilde{c}_{k}\right\}_{k \in \mathbb{Z}}$ satisfies (H1). The following assertions are equivalent:

(i) $\left\{\frac{\alpha \mathrm{i} k-k^{2}}{1+\tilde{c}_{k}}\right\}_{k \in \mathbb{Z}} \subset \rho(A)$ and $\sup _{k}\left\|\frac{-k^{2}}{1+\tilde{c}_{k}}\left(\frac{\alpha \mathrm{i} k-k^{2}}{1+\tilde{c}_{k}}-A\right)^{-1}\right\|<\infty$;

(ii) for every $f \in C^{s}(\mathbb{T} ; X)$, there exists a unique strong $C^{s}$-solution of (1.1) such that $u^{\prime \prime}, u^{\prime}, A u \in C^{s}(\mathbb{T} ; X)$. 
Remark 3.8. Setting $\alpha=0$ and $c=0$ in equation (1.1) we obtain the second-order problem with periodic boundary conditions

$$
\left.\begin{array}{rl}
u^{\prime \prime}(t) & =A u(t)+f(t), \quad 0 \leqslant t \leqslant 2 \pi, \\
u(0) & =u(2 \pi), \\
u^{\prime}(0) & =u^{\prime}(2 \pi),
\end{array}\right\}
$$

and we may apply Theorem 3.6 to obtain a necessary and sufficient condition in order for such a problem to have maximal regularity in Besov spaces. In [2] Arendt and Bu studied the problem (3.7) for $A$ a closed linear operator defined on Banach space $X$ with the unconditional martingale difference property. They established conditions for maximal regularity in $L_{2 \pi}^{p}(\mathbb{R} ; X)$ in terms of $R$-boundedness. In [3], the authors obtained maximal regularity for (3.7) in periodic vector-valued Besov spaces.

\section{The resonance case}

We define

$$
\rho_{d, e}(A)=\left\{\lambda \in \mathbb{C}: d(\lambda) I-e(\lambda) A \text { is invertible and }(d(\lambda)-e(\lambda) A)^{-1} \in \mathcal{B}(X,[D(A)])\right\} .
$$

In what follows we will assume that $d(\mathrm{i} k)$ and $e(\mathrm{i} k)$ exist for all $k \in \mathbb{Z}$. We suppose that $\lambda \rightarrow d(\lambda)$ (respectively, $e(\lambda)$ ) admits an analytical extension to a sector containing the imaginary axis, and still denote this extension by $d$ (respectively, $e$ ).

Denote by $\sigma_{d, e}(A)$ the complementary set $\mathbb{C} \backslash \rho_{d, e}(A)$.

Now, we consider a resonance case. We assume that there are $k_{1}, \ldots, k_{N} \in \mathbb{Z}$ such that

$$
\begin{array}{cl}
\mathrm{i} k_{j} \in \sigma_{d, e}(A) & \text { for } j=1, \ldots, N, \\
\mathrm{i} k \notin \sigma_{d, e}(A) & \text { for } k \in \mathbb{Z}, k \neq k_{1}, \ldots \\
\mathrm{i} k_{j} \text { is a simple pole of } F(\cdot) & \text { for } j=1, \ldots, N,
\end{array}
$$

where $F: \rho_{d, e}(A) \subset \mathbb{C} \rightarrow \mathcal{B}(X,[D(A)])$ is defined by $F(\lambda)=(d(\lambda) I-e(\lambda) A)^{-1}$.

We now give some preliminary results about the solvability of the equation

$$
\left(d\left(\lambda_{0}\right) I-e\left(\lambda_{0}\right) A\right) x=y
$$

where $\lambda_{0}$ is a simple pole of $F(\cdot)$.

We denote by $Q$ the residue of $F(\cdot)$ at $\lambda_{0}$, that is,

$$
Q=\lim _{\lambda \rightarrow \lambda_{0}}\left(\lambda-\lambda_{0}\right) F(\lambda)=\frac{1}{2 \pi \mathrm{i}} \int_{B\left(\lambda_{0}, \varepsilon\right)} F(\lambda) \mathrm{d} \lambda,
$$

where $\varepsilon>0$ and $B\left(\lambda_{0}, \varepsilon\right):=\left\{\lambda \in \mathbb{C}:\left|\lambda-\lambda_{0}\right|<\varepsilon\right\}$.

We define

$$
G(\lambda)= \begin{cases}\left(\lambda-\lambda_{0}\right) F(\lambda), & 0<\left|\lambda-\lambda_{0}\right|<\varepsilon, \\ Q, & \lambda=\lambda_{0} .\end{cases}
$$

We note that $Q \in \mathcal{B}(X,[D(A)])$ is a non-zero operator which verifies the following property. 
Lemma 4.1. With the notation as above, we have

$$
Q=Q\left[d^{\prime}\left(\lambda_{0}\right) I-e^{\prime}\left(\lambda_{0}\right) A\right] Q .
$$

Proof. For each $\lambda$, $\mu$ belonging to $B\left(\lambda_{0}, \varepsilon\right) \backslash\left\{\lambda_{0}\right\}$ with $\left|\lambda-\lambda_{0}\right|>\left|\mu-\lambda_{0}\right|$ we have

$$
\begin{aligned}
F(\lambda)-F(\mu) & =F(\lambda)[d(\mu) I-e(\mu) A-d(\lambda) I+e(\lambda) A] F(\mu) \\
& =F(\lambda)[(d(\mu)-d(\lambda)) I+(e(\lambda)-e(\mu)) A] F(\mu) .
\end{aligned}
$$

Hence,

$$
\frac{F(\lambda)-F(\mu)}{\lambda-\mu}\left(\lambda-\lambda_{0}\right)\left(\mu-\lambda_{0}\right)=\left(\lambda-\lambda_{0}\right) F(\lambda)\left[\frac{d(\mu)-d(\lambda)}{\lambda-\mu} I+\frac{e(\lambda)-e(\mu)}{\lambda-\mu} A\right]\left(\mu-\lambda_{0}\right) F(\mu)
$$

and, using (4.4), we have

$$
G(\lambda) \frac{\mu-\lambda_{0}}{\lambda-\mu}-G(\mu) \frac{\lambda-\lambda_{0}}{\lambda-\mu}=G(\lambda)\left[\frac{d(\mu)-d(\lambda)}{\lambda-\mu} I+\frac{e(\lambda)-e(\mu)}{\lambda-\mu} A\right] G(\mu) .
$$

Since $A \in \mathcal{B}([D(A)], X)$, letting $\mu \rightarrow \lambda_{0}$, we obtain

$$
-Q=G(\lambda)\left[\frac{d\left(\lambda_{0}\right)-d(\lambda)}{\lambda-\lambda_{0}} I+\frac{e(\lambda)-e\left(\lambda_{0}\right)}{\lambda-\lambda_{0}} A\right] Q .
$$

Letting $\lambda \rightarrow \lambda_{0}$, we get

$$
Q=Q\left[d^{\prime}\left(\lambda_{0}\right) I-e^{\prime}\left(\lambda_{0}\right) A\right] Q
$$

This proves the lemma.

The following result is the key for results on the existence of solutions in the resonance case.

Proposition 4.2. Let $\lambda_{0}$ be a simple pole of $F(\cdot)$ and let $Q \in \mathcal{B}(X,[D(A)])$ be defined by (4.3). Then

$$
\operatorname{Ker}\left(d\left(\lambda_{0}\right) I-e\left(\lambda_{0}\right) A\right)=Q(X) .
$$

Moreover, for any $y \in X$ such that $Q y=0$, all solutions of (4.2) are given by

$$
x=G^{\prime}\left(\lambda_{0}\right) y-Q A\left(e^{\prime} G\right)^{\prime}\left(\lambda_{0}\right) y+Q\left(d^{\prime} G\right)^{\prime}\left(\lambda_{0}\right) y .
$$

Proof. First we prove (4.5). For any sufficiently small $\varepsilon>0$ and $0<\left|\lambda-\lambda_{0}\right|<\varepsilon$ we have

$$
\begin{aligned}
\left(d\left(\lambda_{0}\right) I-e\left(\lambda_{0}\right) A\right) G(\lambda) & =\left(\lambda-\lambda_{0}\right)-(d(\lambda) I-e(\lambda) A) G(\lambda)+\left(d\left(\lambda_{0}\right) I-e\left(\lambda_{0}\right) A\right) G(\lambda) \\
& =\left(\lambda-\lambda_{0}\right)+\left(d\left(\lambda_{0}\right)-d(\lambda)\right) G(\lambda)+\left(e(\lambda)-e\left(\lambda_{0}\right)\right) A G(\lambda) .
\end{aligned}
$$

Since $A \in \mathcal{B}([D(A)], X)$, letting $\lambda \rightarrow \lambda_{0}$, we obtain $\left(d\left(\lambda_{0}\right) I-e\left(\lambda_{0}\right) A\right) Q=0$, so that $Q(X)$ is contained in $\operatorname{Ker}\left(d\left(\lambda_{0}\right) I-e\left(\lambda_{0}\right) A\right)$. Now let $x \in D(A)$ be such that $\left(d\left(\lambda_{0}\right) I-e\left(\lambda_{0}\right) A\right) x=$ 0 . Then, for $0<\left|\lambda-\lambda_{0}\right|<\varepsilon$ with $\varepsilon$ small, we have

$$
F(\lambda)\left(d\left(\lambda_{0}\right) I-e\left(\lambda_{0}\right) A\right) x=0 .
$$


For each $x \in X$ we have the identity $x-F(\lambda)(d(\lambda) I-e(\lambda) A) x=0$ or, equivalently,

$$
x+F(\lambda)\left[d\left(\lambda_{0}\right)-d(\lambda)\right] x+F(\lambda)\left[e(\lambda)-e\left(\lambda_{0}\right)\right] A x-F(\lambda)\left[d\left(\lambda_{0}\right) I-e\left(\lambda_{0}\right) A\right] x=0 .
$$

It follows from (4.7) that

$$
x-\left(\lambda-\lambda_{0}\right) F(\lambda) \frac{d(\lambda)-d\left(\lambda_{0}\right)}{\lambda-\lambda_{0}} x+\left(\lambda-\lambda_{0}\right) F(\lambda) \frac{e(\lambda)-e\left(\lambda_{0}\right)}{\lambda-\lambda_{0}} A x=0,
$$

that is, using (4.4),

$$
x-G(\lambda) \frac{d(\lambda)-d\left(\lambda_{0}\right)}{\lambda-\lambda_{0}} x+G(\lambda) \frac{e(\lambda)-e\left(\lambda_{0}\right)}{\lambda-\lambda_{0}} A x=0 .
$$

Letting $\lambda \rightarrow \lambda_{0}$, we get

$$
x-Q d^{\prime}\left(\lambda_{0}\right) x+Q e^{\prime}\left(\lambda_{0}\right) A x=0,
$$

so that $x$ belongs to $Q(X)$, proving (4.5).

Let us now prove (4.6). First we claim that

$$
\lim _{\lambda \rightarrow \lambda_{0}} F(\lambda)\left[I+\left(e^{\prime}\left(\lambda_{0}\right) A-d^{\prime}\left(\lambda_{0}\right) I\right) Q\right]=G^{\prime}\left(\lambda_{0}\right)-Q A\left(e^{\prime} G\right)^{\prime}\left(\lambda_{0}\right)+Q\left(d^{\prime} G\right)^{\prime}\left(\lambda_{0}\right) .
$$

In fact,

$$
\begin{aligned}
G^{\prime}(\lambda)= & F(\lambda)-\left(\lambda-\lambda_{0}\right) F(\lambda)\left[d^{\prime}(\lambda) I-e^{\prime}(\lambda) A\right] F(\lambda) \\
= & F(\lambda)-\left(\lambda-\lambda_{0}\right) F(\lambda) d^{\prime}(\lambda) F(\lambda)+\left(\lambda-\lambda_{0}\right) F(\lambda) e^{\prime}(\lambda) A F(\lambda) \\
= & F(\lambda)\left[I+\left(e^{\prime}\left(\lambda_{0}\right) A-d^{\prime}\left(\lambda_{0}\right) I\right) Q\right]-F(\lambda) e^{\prime}\left(\lambda_{0}\right) A Q+F(\lambda) d^{\prime}\left(\lambda_{0}\right) Q \\
& \quad-F(\lambda) d^{\prime}(\lambda)\left(\lambda-\lambda_{0}\right) F(\lambda)+F(\lambda) e^{\prime}(\lambda) A\left(\lambda-\lambda_{0}\right) F(\lambda) \\
= & F(\lambda)\left[I+\left(e^{\prime}\left(\lambda_{0}\right) A-d^{\prime}\left(\lambda_{0}\right) I\right) Q\right]+F(\lambda) A\left[e^{\prime}(\lambda) G(\lambda)-e^{\prime}\left(\lambda_{0}\right) Q\right] \\
& \quad-F(\lambda)\left[d^{\prime}(\lambda) G(\lambda)-d^{\prime}\left(\lambda_{0}\right) Q\right] \\
= & F(\lambda)\left[I+\left(e^{\prime}\left(\lambda_{0}\right) A-d^{\prime}\left(\lambda_{0}\right) I\right) Q\right]+\left(\lambda-\lambda_{0}\right) F(\lambda) A\left[\frac{e^{\prime}(\lambda) G(\lambda)-e^{\prime}\left(\lambda_{0}\right) Q}{\lambda-\lambda_{0}}\right] \\
& \quad-\left(\lambda-\lambda_{0}\right) F(\lambda)\left[\frac{d^{\prime}(\lambda) G(\lambda)-d^{\prime}\left(\lambda_{0}\right) Q}{\lambda-\lambda_{0}}\right] .
\end{aligned}
$$

Since $A \in \mathcal{B}([D(A)], X)$, letting $\lambda \rightarrow \lambda_{0}$ in the above identity we obtain the claim.

On the other hand, using Lemma 4.1 we obtain

$$
\begin{aligned}
& \lim _{\lambda \rightarrow \lambda_{0}}[\left.d\left(\lambda_{0}\right) I-e\left(\lambda_{0}\right) A\right] F(\lambda)\left[I+\left(e^{\prime}\left(\lambda_{0}\right) A-d^{\prime}\left(\lambda_{0}\right) I\right) Q\right] \\
&= \lim _{\lambda \rightarrow \lambda_{0}}\left[d(\lambda) I-e(\lambda) A+e(\lambda) A-d(\lambda) I+d\left(\lambda_{0}\right) I-e\left(\lambda_{0}\right) A\right] \\
& \times F(\lambda)\left[I+\left(e^{\prime}\left(\lambda_{0}\right) A-d^{\prime}\left(\lambda_{0}\right) I\right) Q\right] \\
&= \lim _{\lambda \rightarrow \lambda_{0}}\left[I+\left\{\frac{e(\lambda)-e\left(\lambda_{0}\right)}{\lambda-\lambda_{0}} A-\frac{d(\lambda)-d\left(\lambda_{0}\right)}{\lambda-\lambda_{0}} I\right\}\left(\lambda-\lambda_{0}\right) F(\lambda)\right] \\
& \quad \times\left[I+\left(e^{\prime}\left(\lambda_{0}\right) A-d^{\prime}\left(\lambda_{0}\right) I\right) Q\right]
\end{aligned}
$$




$$
\begin{aligned}
& =\left[I+\left(e^{\prime}\left(\lambda_{0}\right) A-d^{\prime}\left(\lambda_{0}\right) I\right) Q\right]\left[I+\left(e^{\prime}\left(\lambda_{0}\right) A-d^{\prime}\left(\lambda_{0}\right) I\right) Q\right] \\
& =I+2\left(e^{\prime}\left(\lambda_{0}\right) A-d^{\prime}\left(\lambda_{0}\right) I\right) Q+\left(e^{\prime}\left(\lambda_{0}\right) A-d^{\prime}\left(\lambda_{0}\right) I\right) Q\left(e^{\prime}\left(\lambda_{0}\right) A-d^{\prime}\left(\lambda_{0}\right) I\right) Q \\
& =I+2\left(e^{\prime}\left(\lambda_{0}\right) A-d^{\prime}\left(\lambda_{0}\right) I\right) Q-\left(e^{\prime}\left(\lambda_{0}\right) A-d^{\prime}\left(\lambda_{0}\right) I\right) Q \\
& =I+\left(e^{\prime}\left(\lambda_{0}\right) A-d^{\prime}\left(\lambda_{0}\right) I\right) Q .
\end{aligned}
$$

Due to (4.8) and the fact that $A$ belongs to $\mathcal{B}([D(A)], X)$ we have

$$
\left[d\left(\lambda_{0}\right)-e\left(\lambda_{0}\right) A\right]\left[G^{\prime}\left(\lambda_{0}\right)-Q A\left(e^{\prime} G\right)^{\prime}\left(\lambda_{0}\right)+Q\left(d^{\prime} G\right)^{\prime}\left(\lambda_{0}\right)\right]=I+\left(e^{\prime}\left(\lambda_{0}\right) A-d^{\prime}\left(\lambda_{0}\right) I\right) Q .
$$

Therefore, if $y \in X$ is such that $Q y=0$, equation (4.2) is solvable, and the solution is given by

$$
w=G^{\prime}\left(\lambda_{0}\right) y-Q A\left(e^{\prime} G\right)^{\prime}\left(\lambda_{0}\right) y+Q\left(d^{\prime} G\right)^{\prime}\left(\lambda_{0}\right) y .
$$

Now, arguing as in the proof of Theorem 3.6, we find that, if $f \in B_{p, q}^{s}(\mathbb{T} ; X)$ and $u \in B_{p, q}^{s+2}(\mathbb{T} ; X)$ is a strong $B_{p, q}^{s}$-solution of $(1.1)$, then

$$
\left(-k^{2}+\alpha \mathrm{i} k-\left(1+\tilde{c}_{k}\right) A\right) \hat{u}(k)=\hat{f}(k), \quad k \in \mathbb{Z} .
$$

We suppose that $\lambda \rightarrow \tilde{c}(\lambda)$ admits an analytical extension to a sector containing the imaginary axis, and still denote this extension by $\tilde{c}$.

Substituting $d(\lambda):=\lambda^{2}+\alpha \lambda$ and $e(\lambda):=1+\tilde{c}(\lambda)$, we have

$$
F(\lambda)=\left(\lambda^{2}+\alpha \lambda-(1+\tilde{c}(\lambda)) A\right)^{-1} \quad \text { for all } \lambda \in \rho_{d, e}(A) .
$$

Now, we assume that there are $k_{1}, \ldots, k_{N} \in \mathbb{Z}$ such that (4.1) holds.

For each $k \neq k_{n}, n=1, \ldots, N$, equation (4.10) can be uniquely solved, with

$$
\hat{u}(k)=\left(-k^{2}+\alpha \mathrm{i} k-\left(1+\tilde{c}_{k}\right) A\right)^{-1} \hat{f}(k) .
$$

For $k_{n}, n=1, \ldots, N$, by Proposition 4.2, equation (4.10) is solvable if and only if

$$
Q_{n} \hat{f}\left(k_{n}\right)=0,
$$

where $Q_{n}$ is the residue of $F(\cdot)$ at $\lambda=\mathrm{i} k_{n}$. If (4.11) holds, then, by (4.6), the Fourier coefficients of the solution to (4.10) in $k_{n}, n=1, \ldots, N$ are given by

$$
\hat{u}\left(k_{n}\right)=\left[G_{n}^{\prime}\left(\mathrm{i} k_{n}\right)-Q_{n} A\left(\tilde{c}^{\prime} G_{n}\right)^{\prime}\left(\mathrm{i} k_{n}\right)+Q_{n}\left(d^{\prime} G_{n}\right)^{\prime}\left(\mathrm{i} k_{n}\right)\right] \hat{f}\left(k_{n}\right),
$$

where $G_{n}: B\left(\mathrm{i} k_{n}, \varepsilon\right) \rightarrow \mathcal{B}(X,[D(A)])$ is the analytic function defined by

$$
G_{n}(\lambda)= \begin{cases}\left(\lambda-\mathrm{i} k_{n}\right) F(\lambda), & 0<\left|\lambda-\mathrm{i} k_{n}\right|<\varepsilon \\ Q_{n}, & \lambda=\mathrm{i} k_{n},\end{cases}
$$

for any $\varepsilon>0$ sufficiently small. 
Now, define the family operators

$$
N_{k}= \begin{cases}\left(-k^{2}+\alpha \mathrm{i} k-\left(1+\tilde{c}_{k}\right) A\right)^{-1}, & k \in \mathbb{Z} \backslash\left\{k_{1}, \ldots, k_{N}\right\}, \\ G_{j}^{\prime}\left(\mathrm{i} k_{j}\right)-Q_{j} A\left(\tilde{c}^{\prime} G_{j}\right)^{\prime}\left(\mathrm{i} k_{j}\right)+Q_{j}\left(d^{\prime} G_{j}\right)^{\prime}\left(\mathrm{i} k_{j}\right), & j=1, \ldots, N,\end{cases}
$$

where $\mathrm{i} k \in \rho_{d, e}(A)$ for all $k \in \mathbb{Z} \backslash\left\{k_{1}, \ldots, k_{N}\right\}$. Note that $\left\{N_{k}\right\}_{k \in \mathbb{Z}} \subset \mathcal{B}(X)$.

The following main theorem gives compatibility conditions on $f$ that are necessary and sufficient for the existence of a strong $L^{p}$-solution of (1.1).

Theorem 4.3. Let $1 \leqslant p, q \leqslant \infty$ and let $s>0$. Let $c \in L^{1}\left(\mathbb{R}_{+}\right)$function such that the Laplace transform $\tilde{c}_{k}$ satisfies (H1). Suppose that (4.1) holds. Let $A$ be a closed linear operator defined on a Banach space $X$. If $\sup _{k \in \mathbb{Z}}\left\|k^{2} N_{k}\right\|<\infty$ is bounded, then, for every $f \in B_{p, q}^{s}(\mathbb{T} ; X)$, equation (1.1) has a strong $B_{p, q}^{s}$-solution if and only if $Q_{n} \hat{f}\left(k_{n}\right)=0$, for every $n=1, \ldots, N$.

In this case, all the strong solutions of (1.1) are given by

$$
\begin{aligned}
u(t)=\lim _{n \rightarrow \infty} \sum_{\substack{k=-n, k_{1} \\
k \neq k_{1}, \ldots, k_{N}}}^{n}\left(1-\frac{|k|}{n+1}\right) \mathrm{e}^{\mathrm{i} k t}\left(-k^{2}+\alpha \mathrm{i} k-\left(1+\tilde{c}_{k}\right) A\right)^{-1} \hat{f}(k) \\
\quad+\sum_{j=1}^{N} \mathrm{e}^{\mathrm{i} k_{j} t}\left[G_{j}^{\prime}\left(\mathrm{i} k_{j}\right)-Q_{j} A\left(\tilde{c}^{\prime} G_{j}\right)^{\prime}\left(\mathrm{i} k_{j}\right)+Q_{j}\left(d^{\prime} G_{j}\right)^{\prime}\left(\mathrm{i} k_{j}\right)\right] \hat{f}\left(k_{j}\right) .
\end{aligned}
$$

Proof. First we assume that, for every $f \in B_{p, q}^{s}(\mathbb{T} ; X)$, there exists $v \in B_{p, q}^{s+2}(\mathbb{T} ; X)$, a strong $B_{p, q}^{s}$-solution of the equation (1.1). Taking Fourier transforms on both sides of (1.1), we find that $\hat{v}(k) \in D(A)$ and

$$
\left(-k^{2}+\alpha \mathrm{i} k-\left(1+\tilde{c}_{k}\right) A\right) \hat{v}(k)=\hat{f}(k) \quad \text { for all } k \in \mathbb{Z} .
$$

For $\lambda \in \rho_{d, e}(A)$ and $k_{1}, k_{2}, \ldots, k_{N}$, we have

$$
\left(\lambda-\mathrm{i} k_{j}\right) F(\lambda)\left[\lambda^{2}+\alpha \lambda-(1+\tilde{c}(\lambda)) A\right] \hat{v}\left(k_{j}\right)=\left(\lambda-\mathrm{i} k_{j}\right) \hat{v}\left(k_{j}\right) .
$$

Setting $\lambda \rightarrow \mathrm{i} k_{j}$ it follows that

$$
\lim _{\lambda \rightarrow \mathrm{i} k_{j}}\left(\lambda-\mathrm{i} k_{j}\right) F(\lambda)\left[\lambda^{2}+\alpha \lambda-(1+\tilde{c}(\lambda)) A\right] \hat{v}\left(k_{j}\right)=0 .
$$

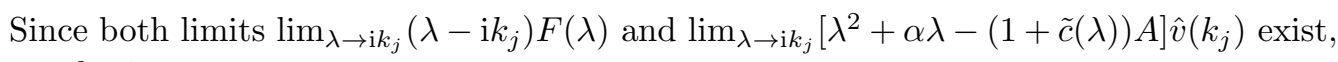
we obtain

$$
Q_{j}\left(-k_{j}^{2}+\alpha \mathrm{i} k_{j}-\left(1+\tilde{c}\left(\mathrm{i} k_{j}\right)\right) A\right) \hat{v}\left(k_{j}\right)=0,
$$

or, equivalently, $Q_{j} \hat{f}\left(k_{j}\right)=0$, for all $k_{j}, j=1, \ldots, N$. Hence, by Proposition 4.2, equation (4.10) is solvable and

$$
\hat{v}(k)= \begin{cases}\left(-k^{2}+\alpha \mathrm{i} k-\left(1+\tilde{c}_{k}\right) A\right)^{-1} \hat{f}(k), & k \in \mathbb{Z} \backslash\left\{k_{1}, \ldots, k_{N}\right\}, \\ {\left[G_{j}^{\prime}\left(\mathrm{i} k_{j}\right)-Q_{j} A\left(\tilde{c}^{\prime} G_{j}\right)^{\prime}\left(\mathrm{i} k_{j}\right)+Q_{j}\left(d^{\prime} G_{j}\right)^{\prime}\left(\mathrm{i} k_{j}\right)\right] \hat{f}(k),} & j=1, \ldots, N,\end{cases}
$$

from which (4.15) follows. 
Conversely, assume that $f \in B_{p, q}^{s}(\mathbb{T} ; X)$ and $Q_{n} \hat{f}\left(k_{n}\right)=0$ for $n=1, \ldots, N$. We define $u(t)$ by (4.15). Then

$$
\hat{u}(k)=N_{k} \hat{f}(k)
$$

for all $k \in \mathbb{Z}$, where $N_{k}$ is defined by (4.14). Note that $\hat{u}(k) \in D(A)$ for all $k \in \mathbb{Z}$.

For each $k \in \mathbb{Z}$, we define $M_{k}:=-k^{2} N_{k}$. By hypothesis, $\left\{M_{k}\right\}_{k \in \mathbb{Z}}$ is bounded. We observe that $\left\{k\left(M_{k+1}-M_{k}\right)\right\}_{k \in \mathbb{Z}}$ and $\left\{k^{2}\left(M_{k+1}-2 M_{k}+M_{k-1}\right)\right\}_{k \in \mathbb{Z}}$ are bounded, which can be proved following the same method as the proof of Proposition 3.3. Then, by Theorem 2.5, we see that $\left\{M_{k}\right\}_{k \in \mathbb{Z}}$ is a $B_{p q}^{s}$-multiplier.

Analogously to the proof of Theorem 3.6, it follows that the family $\left\{\mathrm{i} k N_{k}\right\}_{k \in \mathbb{Z}}$ is a $B_{p q}^{s}$-multiplier. Hence, there exist $v, w \in B_{p, q}^{s}(\mathbb{T} ; X)$ such that

$$
-k^{2} \hat{u}(k)=\mathrm{i} k \hat{v}(k)=\hat{w}(k) .
$$

By $\left[\mathbf{2}\right.$, Lemma 2.1] and $[\mathbf{2}, \S 6], u, u^{\prime}$ are differentiable a.e. with $u^{\prime}=v, w=v^{\prime}=u^{\prime \prime}$ and $u(0)=u(2 \pi), u^{\prime}(0)=u^{\prime}(2 \pi)$. By $\left[\mathbf{3}\right.$, Theorem 2.3] this implies that $u \in B_{p, q}^{s+2}(\mathbb{T} ; X)$.

Now, we show that $u(t) \in D(A)$. Since $Q_{n} \hat{f}\left(k_{n}\right)=0$ for all $n=1, \ldots, N$, by Proposition 4.2 we have

$$
\left(-k^{2}+\alpha \mathrm{i} k-\left(1+\tilde{c}_{k}\right) A\right) N_{k} \hat{f}(k)=\hat{f}(k)
$$

for all $k \in \mathbb{Z}$, or, equivalently,

$$
\begin{aligned}
A N_{k} \hat{f}(k) & =\frac{-k^{2}+\alpha \mathrm{i} k}{1+\tilde{c}_{k}} N_{k} \hat{f}(k)-\frac{1}{1+\tilde{c}_{k}} \hat{f}(k) \\
& =\frac{1}{1+\tilde{c}_{k}}\left(-k^{2} N_{k}\right) \hat{f}(k)+\frac{\alpha}{1+\tilde{c}_{k}} \mathrm{i} k N_{k} \hat{f}(k)-\frac{1}{1+\tilde{c}_{k}} \hat{f}(k) \\
& =\frac{1}{1+\tilde{c}_{k}} \hat{w}(k)+\frac{\alpha}{1+\tilde{c}_{k}} \hat{v}(k)-\frac{1}{1+\tilde{c}_{k}} \hat{f}(k) .
\end{aligned}
$$

Since $f, v, w \in B_{p, q}^{s}(\mathbb{T} ; X)$ and by Corollary 3.4 the family $\left\{I /\left(1+\tilde{c}_{k}\right)\right\}$ is a $B_{p, q}^{s}$-multiplier, there exists $g \in B_{p, q}^{s}(\mathbb{T} ; X)$ such that

$$
A N_{k} \hat{f}(k)=\hat{g}(k) .
$$

From (4.17) we obtain $A \hat{u}(k)=\hat{g}(k)$. By [2, Lemma 3.1] this implies that $u(t) \in D(A)$. By (4.18) we have

$$
\begin{aligned}
\hat{w}(k) & =-k^{2} \hat{u}(k)=-\alpha \hat{v}(k)+\left[1+\tilde{c}_{k}\right] A N_{k} \hat{f}(k)+\hat{f}(k) \\
& =-\alpha \mathrm{i} k \hat{u}(k)+\left[1+\tilde{c}_{k}\right] A \hat{u}(k)+\hat{f}(k) \\
& =-\alpha \mathrm{i} k \hat{u}(k)+A \hat{u}(k)+\tilde{c}_{k} A \hat{u}(k)+\hat{f}(k) .
\end{aligned}
$$

It follows from the uniqueness theorem of Fourier coefficients that $u(t)$ defined by $(4.15)$ satisfies (1.1) for almost all $t \in[0,2 \pi]$.

Acknowledgements. The author thanks the anonymous referee for careful reading of the manuscript and useful suggestions. 


\section{References}

1. H. Amann, On the strong solvability of the Navier-Stokes equations. J. Math. Fluid. Mech. 2 (2000), 16-98.

2. W. Arendt And S. Bu, The operator-valued Marcinkiewicz multiplier theorem and maximal regularity. Math. Z. 240 (2002), 311-343.

3. W. AREndt And S. Bu, Operator-valued Fourier multiplier on periodic Besov spaces and applications. Proc. Edinb. Math. Soc. 47 (2004), 15-33.

4. W. AREndt, C. BAtty And S. Bu, Fourier multipliers for Hölder continuous functions and maximal regularity. Studia Math. 160 (2004), 23-51.

5. P. L. Butzer And H. Berens, Semi-groups of operators and aproximation, Die Grundlehren der Mathematischen Wissenschaften, Volume 145 (Springer, 1967).

6. J. B. Conway, A course in functional analysis, Graduate Texts in Mathematics, Volume 96, (Springer, 1990).

7. G. DA PRATo ANd A. LunARdi, Solvability on the real line of a class of linear Volterra integrodifferential equations of parabolic type. Ann. Mat. Pura Appl. 150 (1988), 67-117.

8. V. Keyantuo and C. Lizama, Fourier multipliers and integro-differential equations in Banach spaces. J. Lond. Math. Soc. 69 (2004), 737-750.

9. H. J. Schmeisser AND H. TRIEbel, Topics in Fourier analysis and function spaces (Wiley, 1987).

10. H. TRIEBEL, Theory of function spaces. Monographs in Mathematics, Volume 78 (Birkhäuser, 1983).

11. L. WEIS, Operator-valued Fourier multiplier theorems and maximal $L_{p}$-regularity. Math. Ann. 319 (2001), 735-758.

12. L. WEIS, A new approach to maximal $L_{p}$-regularity, Lecture Notes in Pure and Applied Mathematics, Volume 215, pp. 195-214 (Marcel Dekker, 2001). 